\title{
SALIVA - A PROSPECTIVE DIAGNOSTIC TOOL FOR SARS CoV-2
}

Dental Science $\quad$ C

Tarunya CRRI, Meenakshi Ammal Dental College, MAHER, Alapakkam Main road, Janaki Vishwanath* Nagar, Maduravoyal, Chennai-600095. ${ }^{*}$ Corresponding Author

Shalini Arumugam CRRI, Meenakshi Ammal Dental College, MAHER, Alapakkam Main road, Janaki Nagar, Maduravoyal, Chennai-600095

\section{Dr. Sathish.R}

Assistant Professor, Meenakshi Ammal Dental College, MAHER, Alapakkam Main road, Janaki Nagar, Maduravoyal, Chennai-600095

\begin{tabular}{ll}
\hline $\begin{array}{l}\text { Dr. Anitha } \\
\text { Logaranjini }\end{array}$ & $\begin{array}{l}\text { Associate Professor, Meenakshi Ammal Dental College, MAHER, Alapakkam Main road, } \\
\text { Janaki Nagar, Maduravoyal, Chennai-600095 }\end{array}$ \\
\hline Dr. Prashanthi & $\begin{array}{l}\text { Assistant Professor, Meenakshi Ammal Dental College, MAHER, Alapakkam Main road, } \\
\text { Janaki Nagar, Maduravoyal, Chennai-600095 }\end{array}$ \\
\hline
\end{tabular}

\section{ABSTRACT}

Coronavirus disease 2019 (COVID-19) now called the severe acute respiratory syndrome coronavirus 2 (SARS CoV-2) was first identified in Wuhan city, Hubei province, China. Currently RT-PCR using nasopharyngeal and Oropharyngeal swabs is supposed to be the most efficacious and standard technique for COVID testing, but are invasive and have a few drawbacks due to which, saliva as a diagnostic specimen is proposed. Salivary specimen collection is non-invasive and can be self-collected at any time and repeatedly and greatly minimises the exposure of. healthcare workers to COVID-19.

\section{KEYWORDS}

COVID-19, Saliva, nasopharyngeal swab, oropharyngeal swab, SARS CoV-2

\section{INTRODUCTION}

Coronavirus disease 2019 (COVID-19) now called the severe acute respiratory syndrome coronavirus 2 (SARS CoV-2) was first identified in Wuhan city, Hubei province, China. It was originally reported to the WHO on December 31, 2019 and was labelled a global health emergency on January 30, 2020. The WHO further declared it a global pandemic on March 11, 2020. In response to the current emergency situation, the need for accurate and rapid diagnostic methods is the need of the hour in controlling and effectively treating the outbreak [1].

Currently RT-PCR using nasopharyngeal and Oropharyngeal swabs is supposed to be the most efficacious and standard technique for COVID testing. Oropharyngeal and Nasopharyngeal swabs are the recommended specimens for diagnostic testing, drawbacks of which are it causes a certain degree of discomfort to the patient and is invasive. Based on these drawbacks, a safe alternative and non invasive collection of specimens proposed is the collection of saliva samples, wherein if the sample is self collected by the patient in a sterile container it can greatly decrease the risk of transmission to healthcare workers [2].

SARS CoV-2 has 3 routes by which it appears in salivary secretions. These three routes are:

A. When present in the upper and lower respiratory tract it can reach the oral cavity via droplets.

B. When present in the blood it can present itself through the GCF

C. And when present in major or minor salivary glands it can be released into the saliva via the salivary ducts $[3,5]$.

\section{SALIVAAS A DIAGNOSTIC TOOL FOR SARS COV-2}

The field of salivary studies is growing rapidly and the concept of "salivaomics" emerged. Salivaomics involves analytics pertaining to five major diagnostic markers namely proteins, messenger RNAs, micro-RNAs, metabolic compounds and microbes which offers substantial advantages for diagnostics since states of disease may be associated with detectable changes in the markers, with the only limitation being low concentration of analytes compared to those in blood, to overcome this limitation the development of a precise molecular method is required [4].

As of today, three propositions have been described to collect saliva for COVID testing - coughing out a sample, salivary swabs and collection of saliva directly from the ducts [3].
To et al, in their study in 2020 showed that SARS-CoV-2 could be detected in the salivary specimens of 11 out of 12 patients correlated with the nasopharyngeal swab [6]. Serial salivary specimens showed declination in salivary levels of SARS-CoV-2 RNA after hospitalisation. Viral culture demonstrated that live viruses were present in the saliva of 3 patients. Also, in all the patients whose Nasopharyngeal specimens tested negative for 2019-nCoV, all saliva specimens also tested negative. The use of saliva is preferred over NP or OP specimens for serial viral load monitoring because this would reduce the discomfort to the patient and reduce the health hazards to healthcare workers during repeated sampling. This suggests that COVID-19 transmitted by asymptomatic infection may originate from infected saliva.

Another study by To et al in 2020 used self-collected saliva mixed with nasopharyngeal and bronchopulmonary secretions from deep throat by coughing out in the morning, from confirmed COVID-19 patients [7]. Among 23 COVID-19 patients, 20 patient's salivary specimen showed detectable SARS-CoV-2 RNA. In the temporal profile of viral load, saliva reached the peak viral load during the first week of symptom onset and then declined. It has also been shown that saliva has a high consistency rate of greater than $90 \%$ compared to Nasopharyngeal specimens in the detection of respiratory viruses. Saliva specimens have high sensitivity and specificity by an automated multiplex clinical laboratory improvement amendments-waived point-of-care molecular assay when compared with NP aspirate specimens. Lower respiratory tract sputum is produced by only $28 \%$ of COVID- 19 patients, which indicates a strong limitation of pharyngeal specimen for diagnostic evaluation.

Zhang et al reported that in a group of all positive swabs, most of the positive results at early stage were from oral swabs, while more positive came from anal swabs at late stage of COVID-19, suggesting that oral swabs may indicate early infection of 2019-nCoV but cannot be used as a discharge criteria [8].

Wyllie et al tested self-collected saliva samples from COVID-19 wards and when compared to SARS-CoV-2 detection from patient matched Nasopharyngeal and saliva samples, they found that saliva yielded greater detection sensitivity and consistency throughout the course of infection. Also, saliva of 2 asymptomatic healthcare workers showed presence of SARS-CoV2 compared to their negative matched Nasopharyngeal swabs, suggesting that saliva may also be a viable alternative for identifying mild or subclinical infections. Furthermore, they report less variability in self-sample collection of saliva [9]. 
Azzi et al collected saliva from 25 COVID-19 patients (confirmed by Nasopharyngeal swabs) through the drooling technique and all were tested positive for the presence of SARS-CoV-2, while there was an inverse association between lactate dehydrogenase (LDH) and Cycle threshold $(\mathrm{Ct})$ values. 2 patients showed positive saliva results on the same day when their pharyngeal or respiratory swabs showed conversion. Thus, they conclude that saliva is not only a reliable tool to detect SARS-CoV-2, but also may provide information about the clinical evolution of the disease [10]

\section{PRECEDENCE OF SALIVARY SPECIMENS OVER OTHERS}

Rapid and accurate diagnosis of COVID-19 is vital in containment of the disease in both the hospital and community set-up. Nasopharyngeal and Oropharyngeal swabs are the recommended specimen types for COVID-19 diagnostic testing. The collection of these specimen types requires close contact with the patients, trained healthcare workers, sufficient time and thus a"high risk job." In Nasopharyngeal swabbing patients needs to tilt the head upto 70 degrees to access the Nasopharyngeal region and also may face difficulty if nasal obstructions are present. The swab is kept in place for several seconds to absorb the secretions which increases the risk of sneezing. Oropharyngeal swabbing needs wide mouth opening with tongue depression and chances of patients gagging and coughing during the procedure is high. Both Nasopharyngeal and Oropharyngeal swab procedure can cause bleeding, especially in patients with bleeding diathesis.

There is also a huge need for personal protective equipments (PPE) which can be preserved to be used in critical COVID-19 care wards [3]. Advantages of saliva specimens are that, it can be self-collected easily by adults and children, as the patient spits into a sterile container. Thus, saliva collection will eliminate the need for health care professionals and reduce nosocomial infection. This reduces the time and cost associated with the specimen collection will thus will help in increased testing of patients and in mass screening. Specimen collection can be done where negative ventilation chambers are not available such as in outpatient clinics, community or household areas.

\section{CONCLUSIONS}

Salivary specimen collection is non-invasive and can be self-collected at any time and repeatedly and greatly minimises the exposure of healthcare workers to COVID-19. Furthermore, saliva may also be an appropriate and sensitive bio fluid, as an alternative to Nasopharyngeal and Oropharyngeal swabs for screening asymptomatic or presymptomatic SARS-CoV-2 infections and also desirable for sequential monitoring of viral load. Hence, its concluded that with all the advantages discussed, saliva will emerge as a potential diagnostic specimen for COVID-19 testing.

\section{REFERENCES:}

[1] World Health Organization. Coronavirus. Available at: https:// www, who, int/ emergencies/diseases/novel-coronavirus-2019/global-research-on-novel-coronavirus2019-ncov.Accessed March 2020

[2] World Health Organisation https://www.who.int/health-topics/coronavirus\#tab=tab_1

[3] Harikrishnan P. Saliva as a Potential Diagnostic Specimen for COVID-19 Testing. J Craniofac Surg. 2020 Sep;31(6):e653-e655. doi: 10.1097/SCS.0000000000006724. PMID: 32541273; PMCID: PMC7382405

[4] Shah S. Salivaomics: The current scenario. J Oral and Maxillafac Pathol [serial online] 2018 [cited 2020 Sep 15];22:375-81. Available from: http://www. jomfp. in/text. asp? 2018/22/3/375/247364

[5] Baghizadeh Fini M. Oral saliva and COVID-19. Oral Oncol. 2020 Sep;108:104821. doi: 10.1016/j.oraloncology.2020.104821. Epub 2020 May 27. PMID: 32474389; PMCID: PMC7250788.

[6] To KK, Tsang OT, Yip CC, et al. Consistent detection of 2019 novel coronavirus in saliva. Clin Infect Dis [published ahead of print February 12, 2020] doi: $10.1093 / \mathrm{cid} / \mathrm{ciaa} 149$

[7] To KK, Yip CC, Lai CY,et al.Saliva as a diagnostic specimenfortesting respiratory virus by a point-of-care molecular assay: a diagnostic validity study. Clin Microbiol Infect 2019;25:372-378

[8] ZhangW,DuRH,LiB,etal.Molecularandserological investigation of 2019-nCoV infected patients: implication of multiple shedding routes. Emerg Microbes Infect infected patien

[9] Wyllie AL, Fournier J, Casanovas-Massana A, et al. Saliva is more sensitive for SARSCoV-2 detection in COVID-19 patients than nasopharyngeal swabs. medRxiv [published ahead of print April 22, 2020] doi: 10.1101/2020.04.16.20067835

[10] Azzi L, Carcano G, Gianfagna F, et al. Saliva is a reliable tool to detect SARS-CoV-2. J Infection [published ahead of print April 14, 2020] doi: 10.1016/j.jinf.2020.04.005 